Article

\title{
In Vitro Corrosion and Bioactivity Performance of Surface-Treated Ti-20Nb-13Zr Alloys for Orthopedic Applications
}

\author{
Madhan Kumar Arumugam ${ }^{1, *}$, Mohamed A. Hussein ${ }^{1}\left(\mathbb{D}\right.$, Akeem Yusuf Adesina $^{1}(\mathbb{D}$ and \\ Nasser Al-Aqeeli ${ }^{2, *}$ \\ 1 Center of Research Excellence in Corrosion, King Fahd University of Petroleum and Minerals, \\ Dhahran 31261, Saudi Arabia; mahussein@kfupm.edu.sa (M.A.H.); adesina@kfupm.edu.sa (A.Y.A.) \\ 2 Department of Mechanical Engineering, King Fahd University of Petroleum and Minerals, Dhahran 31261, \\ Saudi Arabia \\ * Correspondence: madhankumar@kfupm.edu.sa (M.K.A.); naqeeli@kfupm.edu.sa (N.A.-A.); \\ Tel.: +96-61-3860-4818 (M.K.A.)
}

Received: 28 March 2019; Accepted: 25 April 2019; Published: 27 May 2019

\begin{abstract}
The influence of surface treatments on the microstructure, in vitro bioactivity and corrosion protection performance of newly fabricated Ti-20Nb-13Zr (TNZ) alloys was evaluated in simulated body fluid (SBF). The TNZ alloy specimens were treated with separate aqueous solutions of $\mathrm{NaOH}$ and $\mathrm{H}_{2} \mathrm{O}_{2}$ and with a mixture of both, followed by thermal treatment. The nanoporous network surface structure observed in $\mathrm{H}_{2} \mathrm{O}_{2}$-treated and alkali-treated specimens was entirely different from the rod-like morphology observed in alkali hydrogen peroxide-treated specimens. XRD results revealed the formation of $\mathrm{TiO}_{2}$ and sodium titanate layers on the TNZ specimens during surface treatments. The water contact angle results implied that the surface-treated specimens exhibited improved surface hydrophilicity, which probably improved the bioactivity of the TNZ specimens. The in vitro corrosion protection performance of the surface-treated TNZ specimens was analyzed using electrochemical corrosion testing in SBF, and the obtained results indicated that the surface-treated specimens exhibited improved corrosion resistance performance compared to that of the bare TNZ specimen. The in vitro bioactivity of the treated TNZ specimens was assessed by soaking in SBF, and all the investigated treated specimens showed numerous apatite nucleation spheres within 3 days of immersion in SBF.
\end{abstract}

Keywords: Ti implants; corrosion; in vitro bioactivity; surface treatment

\section{Introduction}

Metallic materials, including 316L stainless steel (SS), titanium (Ti) and its alloys, and cobalt alloys are employed as orthopedic implants, which are clinically placed inside the human body to restore bone performance through strengthening or substituting an injured bone structure. Mostly, orthopedic implants are utilized as permanent or temporary medical devices depending on the fractured bone. The frequency of orthopedic fractures or illness and the increasing number of aged populations have globally driven the demand for orthopedic implants in recent years. Among the available metallic implant materials, Ti and its alloys have been the most frequently utilized orthopedic implants for the past many decades due to their good ductility, high specific strength, adequate corrosion resistance and acceptable biocompatibility [1-3]. However, the interaction of Ti implants with human bone after implantation is still considered a core concern because Ti cannot interact with bone tissues, which can further influence bone resorption, increasing the possible threat of loosening [4]. Several surface treatments and coatings have been developed in the past decades to create interactions between implants and the surrounding bone and to improve corrosion protection 
performance in the physiological environment [5-7]. Enhanced corrosion and wear resistance and improved osseointegration rates with good biocompatibility are the most significant features that can be achieved through surface modification of orthopedic implants.

The surface features of implant materials play a major role in extending the service life of orthopedic implants. In general, many researchers have enthusiastically explored diverse approaches to achieve biocompatibility and the wear- and corrosion-resistant performance of metallic implants, targeting improved adaptation to the implant environment [8,9]. Hence, the surface treatment approach appears to be the most feasible alternative for enhancing the corrosion resistance and relevant features of biomaterial surfaces. Among the available methods, surface treatment by chemical methods has been widely considered owing to its ease, low cost, bone bonding capability and appropriateness for implants with complex forms $[10,11]$. Sodium hydroxide and hydrogen peroxide have been the most commonly utilized reagents in the surface treatment of Ti implants in recent years [12-14]. Through $\mathrm{NaOH}$ treatment, a sodium titanate film is generated over the Ti substrate; the potential mechanism for the initiation and evolution of hydroxyapatite on surface-modified Ti was reported by Kim et al. [14]. However, Ti alloys produce a titania gel layer with $\mathrm{H}_{2} \mathrm{O}_{2}$ treatment, and these gel layers possess a synergetic effect, inducing hydroxyapatite growth after exposure in simulated body fluid (SBF); hence, treated Ti surfaces are considered bioactive.

Ti-6Al-4V (Ti64/G5) alloys have been the most commonly employed orthopedic implants in the last few decades; however, the leaching of $\mathrm{V}$ and $\mathrm{Al}$ species from the Ti64 implant surface severely disrupts the biocompatibility of orthopedic Ti implants due to the cytotoxicity and neurotoxicity of the released ions, which additionally hinder bone mineralization. Therefore, many Ti-based alloys have been prepared to overcome the shortcomings of Ti64/G5 alloys in the orthopedic field. The author's research team recently developed a nanograined Ti-20Nb-13Zr (TNZ) alloy and investigated the in vitro corrosion resistance and biocompatibility of the developed alloy in SBF medium $[15,16]$. To enhance the in vitro corrosion and bioactivity of TNZ alloy, the target of this investigation is to explore the influence of surface treatment of the newly developed Ti alloy substrates on their corrosion and bioactivity performance in SBF medium.

\section{Materials and Methods}

\subsection{Specimen Preparation}

The base substrate used in the current study is a newly developed TNZ alloy fabricated by ball milling and spark plasma sintering techniques using elemental powders of $\mathrm{Ti}, \mathrm{Nb}$ and $\mathrm{Zr}$ with $99.8 \%$ purity provided by Alfa Aesar, Haverhill, MA, USA, as explained previously [15,17]. TNZ alloy specimen discs with a diameter of $2 \mathrm{~cm}$ were ground using $\mathrm{SiC}$ grit papers from 400 to 2400 and finally polished with alumina to attain a mirror-like surface. Afterward, the specimens were washed with distilled water, ultrasonicated with acetone to remove residuals and then dried in air.

\subsection{Surface Treatment}

First, the TNZ alloy specimens were soaked in an acidic mixture of $\mathrm{HNO}_{3}: \mathrm{HF}: \mathrm{H}_{2} \mathrm{O}$ (1:3:6 vol.) at $60{ }^{\circ} \mathrm{C}$ for $2 \mathrm{~min}$ to remove the natural oxide film; then, the specimen was washed with distilled water for $10 \mathrm{~min}$ and then dried in air. For alkali treatment, the TNZ specimens were immersed in $25 \mathrm{~mL}$ of $10 \mathrm{M} \mathrm{NaOH}$ solution at $60^{\circ} \mathrm{C}$ for $24 \mathrm{~h}$, cleaned with distilled water and dried at $40^{\circ} \mathrm{C}$ for approximately $24 \mathrm{~h}$ in an air furnace. Subsequently, thermal treatment was performed by keeping specimens in an aluminum crucible at $600{ }^{\circ} \mathrm{C}$ for approximately $1 \mathrm{~h}$ in a muffle furnace and allowing them to cool to room temperature (RT) in the furnace. For $\mathrm{H}_{2} \mathrm{O}_{2}$ treatment, TNZ specimens were soaked in $25 \mathrm{~mL}$ of $10 \mathrm{M} \mathrm{H}_{2} \mathrm{O}_{2}$ solution at $80^{\circ} \mathrm{C}$ for $1 \mathrm{~h}$, and then the specimen was thermally treated at $400{ }^{\circ} \mathrm{C}$ for $1 \mathrm{~h} \mathrm{in}$ an air furnace. For alkali-hydrogen peroxide treatment, the TNZ specimens were immersed in $25 \mathrm{~mL}$ of a mixture of $10 \mathrm{M} \mathrm{NaOH}$ and $10 \mathrm{M} \mathrm{H}_{2} \mathrm{O}_{2}$ at $60^{\circ} \mathrm{C}$ for $1 \mathrm{~h}$, and then the specimen was cleaned with distilled water and dried at $40{ }^{\circ} \mathrm{C}$. Subsequently, the specimens were thermally treated at $600{ }^{\circ} \mathrm{C}$ for $1 \mathrm{~h}$ 
and allowed to reach RT in the furnace. Finally, all the treated specimens were ultrasonicated with distilled water to eliminate the attached residues on the specimen surface. The alkali-, $\mathrm{H}_{2} \mathrm{O}_{2}$ - and $\mathrm{NaOH}+\mathrm{H}_{2} \mathrm{O}_{2}$ mixture-treated samples were labeled SHT, HPT and SHPT, respectively.

\subsection{In Vitro Bioactivity of Surface-Treated Samples}

The in vitro bioactivity of orthopedic implants is generally evaluated through the growth of hydroxyapatite on their surface during immersion in a physiological medium. Herein, the in vitro bioactivity of treated TNZ specimens was evaluated based on the procedure in previous reports $[12,13]$. The SBF was prepared according to a previous report [16], and then the samples were immersed in SBF for 3, 5, and 7 days. The growth of hydroxyapatite on treated TNZ specimens was examined after exposure to SBF medium for different immersion periods by SEM/EDX analysis of the treated surface.

\subsection{Characterization}

The surface morphology of the treated specimens was examined using field emission scanning electron microscopy (FE-SEM, Tescan microscope, Brno-Kohoutovice, Czech Republic, accelerated voltage $\sim 20 \mathrm{kV}$ and irradiation current $\sim 10 \mu \mathrm{A}$ ). The microscale roughness of the treated specimen was measured using an optical profilometer (Contour GT-K, Bruker Nano GmbH, Billerica, MA, USA). X-ray diffraction (XRD) patterns of the treated specimen were recorded by an RINT2500 (Rigaku, Tokyo, Japan) with a scattering angle of $20^{\circ}-80^{\circ}$ at a $2 \theta$ step of $0.02^{\circ}$. The water contact angles (WCAs) of treated specimens were monitored using a contact angle meter (VCA OPTIMA, AST Products Inc., Billerica, MA, USA) at five different locations, and the mean was reported. After placing a drop of distilled water $(10 \mu \mathrm{L})$ on the treated specimens, images were snapped instantly. The pictures of drops were analyzed using an image analysis system, and the contact angle (CA) was estimated from the profiles of the drops with a precision of $\pm 0.1^{\circ}$.

\subsection{In Vitro Corrosion-Resistant Behavior of Treated TNZ Specimens}

In vitro corrosion testing was accomplished using a Gamry potentiostat/galvanostat in simulated body fluid medium. Electrochemical corrosion measurements were performed by a conventional three-electrode cell using TNZ specimens as the working electrode and a saturated calomel electrode (SCE) and a graphite rod as the reference and counter electrodes, respectively. The in vitro corrosion test was performed by monitoring the open circuit potential (OCP) for $1 \mathrm{~h}$ to achieve a stable state, followed by linear polarization resistance (LPR), electrochemical impedance spectroscopic (EIS) and, finally, potentiodynamic polarization (PDP) measurements. The LPR test was carried out by applying a potential of $\pm 25 \mathrm{mV}$ from the OCP with a scan rate of $0.125 \mathrm{mV} / \mathrm{s}$. The PDP test was performed in the potential range of -250 to $+1500 \mathrm{mV}$ vs OCP at a scan rate of $0.196 \mathrm{mV} / \mathrm{s}$. The electrochemical impedance spectrum was obtained in the frequency region from $100 \mathrm{kHz}-1 \mathrm{mHz}$ with an amplitude of $10 \mathrm{mV}$ under the open circuit potential. The achieved EIS data were investigated using an equivalent circuit fitted through Echem Analyst software (version 6.25), which was used to calculate the chi-squared $\left(\chi^{2}\right)$ value to evaluate the quality of the equivalent circuit fitting. Moreover, all experiments were repeated three times for consistency.

\section{Results and Discussion}

\subsection{Surface Characterization Results}

SEM micrographs of the treated specimens are shown in Figure 1, and the treated specimens were compared using EDS analysis, shown in Figure S1 in the supporting information. The HPT specimen exhibited a cracked titanium oxide layer, which may occur during the removal of water molecules in thermal treatment. High-magnification SEM images of the HPT specimens showed a porous titania layer with homogeneously networked pores with diameters on the nanometer scale [13]. Porous nanostructures on Ti surfaces play a significant role in implant materials because an interconnected 
open porous structure favors the transportation of body fluid and new bone tissue growth [18]. It has already been reported that the interaction of Ti with $\mathrm{H}_{2} \mathrm{O}_{2}$ yields a TiOOH matrix, which probably facilitates the growth of the $\mathrm{TiO}_{2}$ layer. Furthermore, a narrowly interlinked nanoporous network is formed due to the interaction of Ti with $\mathrm{H}^{+}$ions from the $\mathrm{H}_{2} \mathrm{O}_{2}$ solution, leading to the relocation of the $\mathrm{Ti}$ and $\mathrm{O}$ atoms to form a thermodynamically stable and well-ordered nanostructure [19]. The SHT specimen showed a compact layer with globular-like morphology, and the corresponding EDS results revealed the existence of $\mathrm{Ti}, \mathrm{Nb}, \mathrm{Zr}$, and $\mathrm{O}$, along with $\mathrm{Na}$, which further corroborated the generation of a sodium titanate layer on the TNZ specimen [20]. In general, naturally formed $\mathrm{TiO}_{2}$ films are partially dissolved in $\mathrm{NaOH}$ due to corrosive $\mathrm{OH}^{-}$ions, and the alloy specimens react with $\mathrm{NaOH}$. Furthermore, hydrated $\mathrm{TiO}_{2}$ forms negatively charged hydrates on its surface, which are then combined with sodium ions, finally forming a sodium titanate $\left(\mathrm{Na}_{2} \mathrm{Ti}_{5} \mathrm{O}_{11}\right)$ hydrogel film. The formed hydrogel films were dehydrated and densified to produce stable sodium titanate films during heat treatment [21].
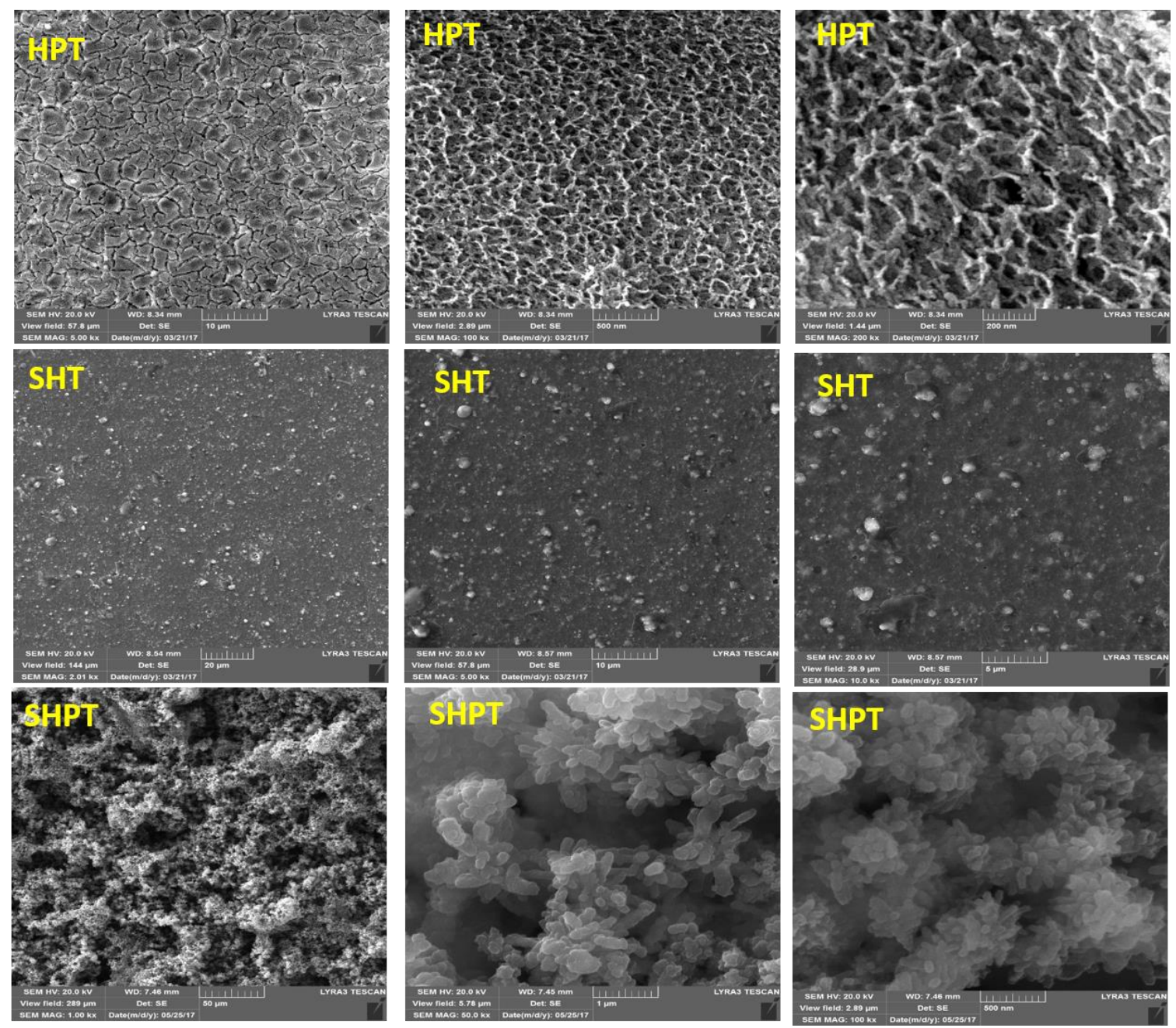

Figure 1. SEM micrographs of treated specimens.

The SHPT specimen showed a rod-like structure with agglomerated particles uniformly distributed throughout its surface. Y. Sasikumar and N. Rajendran have also reported rod-like structures with agglomerated particles on the surface of alkali- and hydrogen peroxide-treated Cp-Ti and Ti-15Mo alloy substrates [22]. It has been established that a porous nanostructure can induce bone bonding ability in the implant surface owing to the higher surface energy and large surface area in the physiological environment [23]. Lin et al. prepared different micro-/nanostructures on Ti implants and reported that 
alkaline hydrothermally treated surfaces with nano-/micro nest-like and nanotube structured surfaces possessed better osseointegration ability [24].

Surface topographic images of bare and treated TNZ specimens are shown in Figure 2. In comparison with the bare TNZ specimen, all the treated specimens revealed higher surface roughness due to the formation of porous/cracked topographic surfaces. HPT specimens showed relatively heterogeneous surface topography with a distribution of pores, whereas SHT and SHPT exhibited comparatively smooth and compact surfaces with few microcracks. Recently, it has been reported that surface microroughness improves osseointegration in the initial stages and in regions of low-quality bone [25]. To obtain clearer information about surface topography, the parameters related to surface roughness were estimated from the optical profilometric images, and the obtained results are summarized in Table S1 in the supporting information. Among the parameters measured, $R_{\mathrm{a}}$ (average surface roughness) and $R_{\mathrm{q}}$ (root mean square, RMS) are considered to be more significant as these parameters indicate the magnitude of deviation from a plain surface determined by scanning a constant surface profile [26]. From Table S1, the $R_{\mathrm{q}}$ and $R_{\mathrm{a}}$ values of SHT and SHPT were lower (between $0.221-3 \mu \mathrm{m}$ ), whereas for the HPT specimen, these values were 10-40 $\mu \mathrm{m}$. The low surface roughness values of the SHT and SHPT specimens implied a homogeneous and more compact layer compared to that of the HPT specimen. The obtained results indicated that the surface roughness parameters were influenced by the different surface modification approaches, and maximum values were observed for the HPT specimen.
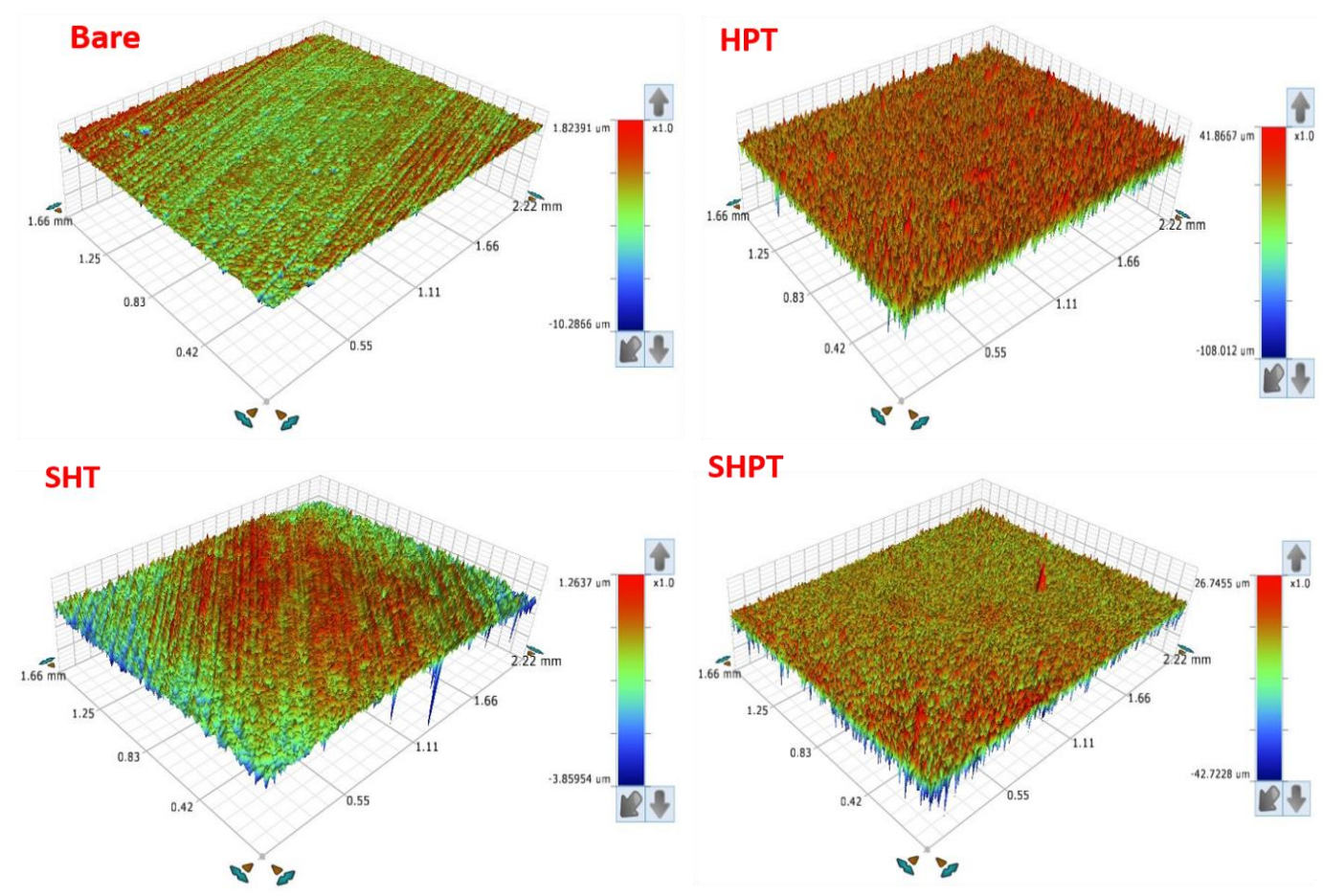

Figure 2. Surface topographic images of treated specimens.

\subsection{XRD Analysis Results}

Figure 3 displays the XRD patterns of surface-treated TNZ specimens. TNZ alloy is classified as a $(\beta+\alpha)$ Ti alloy, as discussed previously [15]. The peaks of the $\beta$ Ti and $\alpha$ Ti phases of TNZ alloy were observed for all treated TNZ specimens. The SHT and SHPT specimens exhibited strong diffraction peaks at $2 \theta$ values of $36.57^{\circ}, 38.25^{\circ}, 42.31^{\circ}$ and $61.6^{\circ}$ (JCPDS No. 00-059-0666), demonstrating the generation of a sodium titanate layer on the TNZ specimens [20]. It is already well known that prismatic sodium titanate has higher crystallinity (because of subsequent thermal treatment) than alkali- and heat-treated Ti alloy [20]. HPT showed several diffraction peaks at $2 \theta=23.41^{\circ}, 37.85^{\circ}$, 


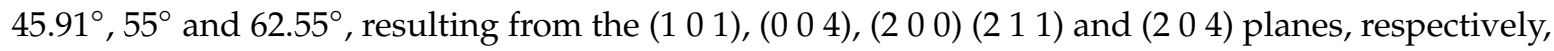
and representing the anatase phase of $\mathrm{TiO}_{2}$. In general, the obtained anatase phase was recognized as a more appropriate phase for the generation of hydroxyapatite than the other phases $[27,28]$.

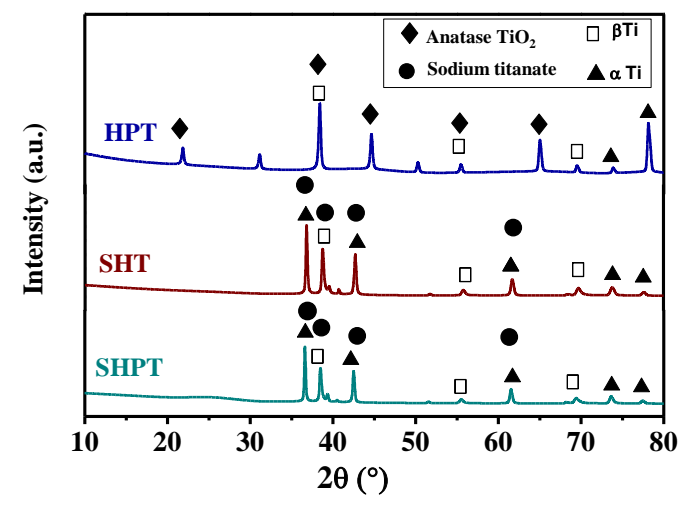

Figure 3. XRD patterns of treated specimens.

\subsection{Contact Angle Results}

Water contact angle (WCA) measurements were utilized to estimate the effect of surface treatment on the surface hydrophilic character of TNZ specimens, and the results are displayed in Figure 4. The WCA of the bare TNZ specimen was observed to be $88.70^{\circ}$, indicating its hydrophobic nature, which is in good agreement with a previous report [29]. However, for the surface-treated TNZ specimens, the obtained WCA values were lower, which confirmed the improved hydrophilicity of the surface-treated TNZ specimens. The SHPT specimen showed the lowest WCA value of $35.30^{\circ}$, which indicated an enhanced hydrophilic surface due to the construction of a sodium titanate film on the TNZ specimen. In general, hydrophilic surfaces on implants facilitate the growth of hydroxyapatite through ion exchange in SBF medium [30]. Pesskova et al. reported that alkali and heat treatment led to a lower contact angle and higher surface free energy by creating more $\mathrm{OH}^{-}$groups on the Ti alloy surface. These features could facilitate the adhesion of necessary proteins and improve cell attachment and proliferation [31]. Herein, the obtained results revealed that surface treatment improves the hydrophilic nature of treated TNZ specimens, which probably enhances their bioactivity.

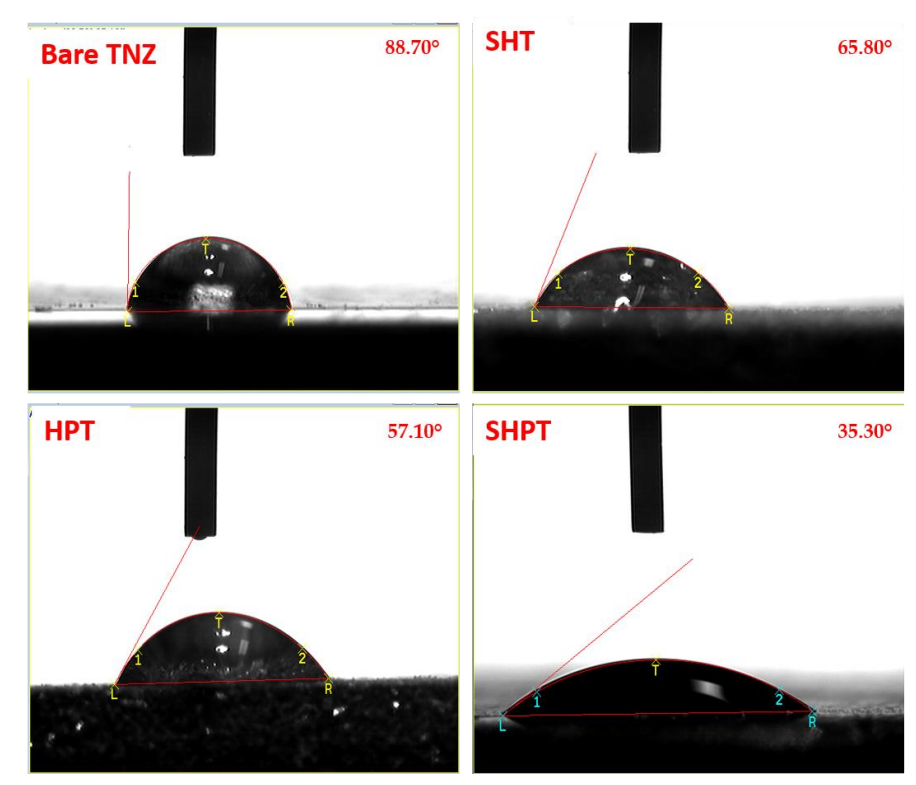

Figure 4. WCA images of treated specimens. 


\subsection{In Vitro Corrosion Resistance Analysis}

Representative PDP curves of bare and treated TNZ specimens in SBF medium are presented in Figure 5. The electrochemical parameters extracted from the PDP curves, including the corrosion current density $\left(I_{\text {corr }}\right)$, corrosion potential $\left(E_{\text {corr }}\right)$, anodic and cathodic slopes $\left(\beta_{\mathrm{a}}\right.$ and $\left.\beta_{\mathrm{c}}\right)$, and passivation current density $\left(I_{\mathrm{p}}\right)$, are summarized in Table 1 .

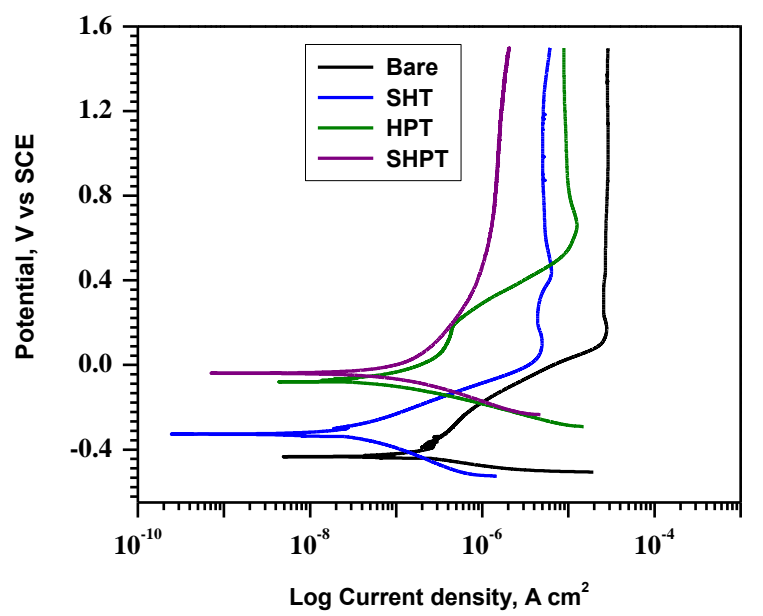

Figure 5. PDP curves of treated specimens in SBF medium.

Table 1. Tafel parameters of bare and treated TNZ specimens.

\begin{tabular}{ccccccc}
\hline Samples & $\boldsymbol{E}_{\text {corr }}(\mathbf{V})$ & $\begin{array}{c}\boldsymbol{I}_{\text {corr }} \\
\left(\boldsymbol{\mu} \mathbf{A} / \mathbf{c m}^{2}\right)\end{array}$ & $\begin{array}{c}\boldsymbol{I}_{\mathbf{p}} \\
\left(\boldsymbol{\mu} \mathbf{A} / \mathbf{c m}^{\mathbf{2}}\right)\end{array}$ & $\begin{array}{c}\boldsymbol{\beta}_{\mathbf{a}} \\
(\mathbf{m V} / \mathbf{d e c})\end{array}$ & $\begin{array}{c}\boldsymbol{\beta}_{\mathbf{c}} \\
(\mathbf{m V} / \mathbf{d e c} .)\end{array}$ & $\begin{array}{c}\text { Corr. Rate } \\
(\mathbf{m m p y}) \times \mathbf{1 0}^{-\mathbf{3}}\end{array}$ \\
\hline Bare & -0.443 & 1.012 & 26.051 & 73 & 92 & 8.880 \\
SHT & -0.331 & 0.125 & 4.812 & 82 & 79 & 1.097 \\
HPT & -0.078 & 0.237 & 9.563 & 74 & 80 & 2.080 \\
SHPT & -0.031 & 0.091 & 1.312 & 89 & 78 & 0.8042 \\
\hline
\end{tabular}

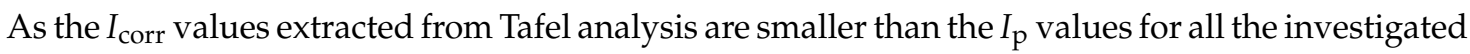
specimens, the TNZ surface is passive at $E_{\text {corr }}$. Hence, the $I_{\text {corr }}$ values are not directly associated with the actual corrosion rates of specimens due to the existence of a passive layer on their surface; therefore, the values summarized in Table 1 can probably be utilized to acquire a comparative ranking of corrosion-resistant behavior [32]. From the observation of the PDP curves, all the investigated specimens exhibited an active-passive transition with a self-passivation region where the increase in current density against potential was nearly circumvented, revealing the formation of a stable passive layer. Generally, if the passivation current density value is lower, the metal surface tends to passivate easily [33]. Comparing the $I_{\mathrm{p}}$ values of bare and treated specimens, bare specimens showed the highest $I_{\mathrm{p}}$ values, followed by the HPT, SHT, and SHPT specimens, which implied the improved corrosion-resistant performance of TNZ specimens after surface treatment. Among the treated specimens, SHPT showed the lowest $I_{\mathrm{p}}$ values, which revealed enhanced passivation behavior in SBF. The $E_{\text {corr }}$ values of TNZ specimens after surface treatment increased, and the $I_{\text {corr }}$ values of TNZ specimens were noticeably lowered after surface treatment, which implied enhanced corrosion resistance in SBF medium.

In particular, the $I_{\text {corr }}$ values of the SHPT specimen were the lowest $\left(0.091 \mu \mathrm{A} / \mathrm{cm}^{2}\right)$ among the treated specimens, indicating that the mixture of $\mathrm{NaOH}$ and $\mathrm{H}_{2} \mathrm{O}_{2}$ solution can provide a more compact sodium titanate layer, which improved the corrosion protection performance in SBF medium.

The LPR curves of bare and treated specimens are displayed in Figure S2 in the supplementary materials. The calculated $I_{\text {corr }}$ and polarization resistance $\left(R_{\mathrm{p}}\right)$ values are summarized in Table 2.

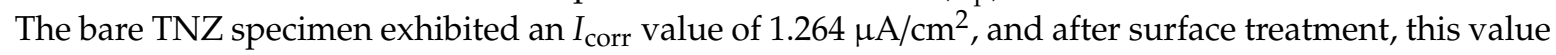


was effectively reduced, which revealed the lower corrosion rate of the treated specimens. Specifically, the $I_{\text {corr }}$ value of SHPT $\left(0.063 \mu \mathrm{A} / \mathrm{cm}^{2}\right)$ was noticeably decreased compared to those for the other treatments, emphasizing the beneficial role of the mixture of $\mathrm{NaOH}$ and $\mathrm{H}_{2} \mathrm{O}_{2}$ in improving the corrosion-resistant performance. In addition, the $R_{\mathrm{p}}$ value for the bare specimen was observed to be $17.67 \mathrm{k} \Omega \cdot \mathrm{cm}^{2}$, whereas for the treated specimens, it was calculated to be in the range of $88-285 \mathrm{k} \Omega \cdot \mathrm{cm}^{2}$. In particular, SHPT specimens showed the highest $R_{\mathrm{p}}$ value, which further corroborated the improved barrier performance of the treated specimens. From the obtained outcomes from PDP and LPR, it was preliminarily inferred that SHPT enhanced the performance of TNZ specimens in SBF medium. To attain significant evidence about the barrier performance of treated TNZ specimens in SBF medium, EIS data were recorded and are displayed in Nyquist and Bode formats in Figures 6 and 7, respectively.

Table 2. LPR parameters of bare and treated TNZ specimens.

\begin{tabular}{ccccc}
\hline Samples & $\boldsymbol{E}_{\text {corr }}(\mathrm{V})$ & $\boldsymbol{I}_{\text {corr }}\left(\boldsymbol{\mu \mathrm { A }} / \mathbf{c m}^{\mathbf{2}}\right)$ & $\boldsymbol{R}_{\mathbf{p}}\left(\mathbf{k} \boldsymbol{\Omega} \cdot \mathbf{c m}^{\mathbf{2}}\right)$ & Corr. Rate $(\mathbf{m m p y}) \times \mathbf{1 0}^{-\mathbf{3}}$ \\
\hline Bare & -0.439 & 1.264 & 17.673 & 11.096 \\
SHT & -0.339 & 0.093 & 187.80 & 0.8164 \\
HPT & -0.069 & 0.189 & 88.313 & 1.654 \\
SHPT & -0.028 & 0.063 & 286.440 & 0.5534 \\
\hline
\end{tabular}

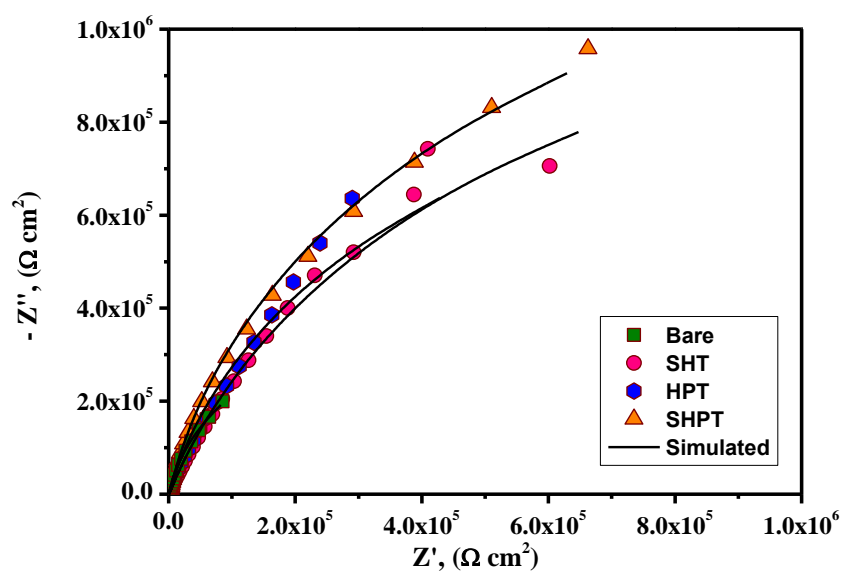

Figure 6. Nyquist curves of treated specimens in SBF medium.

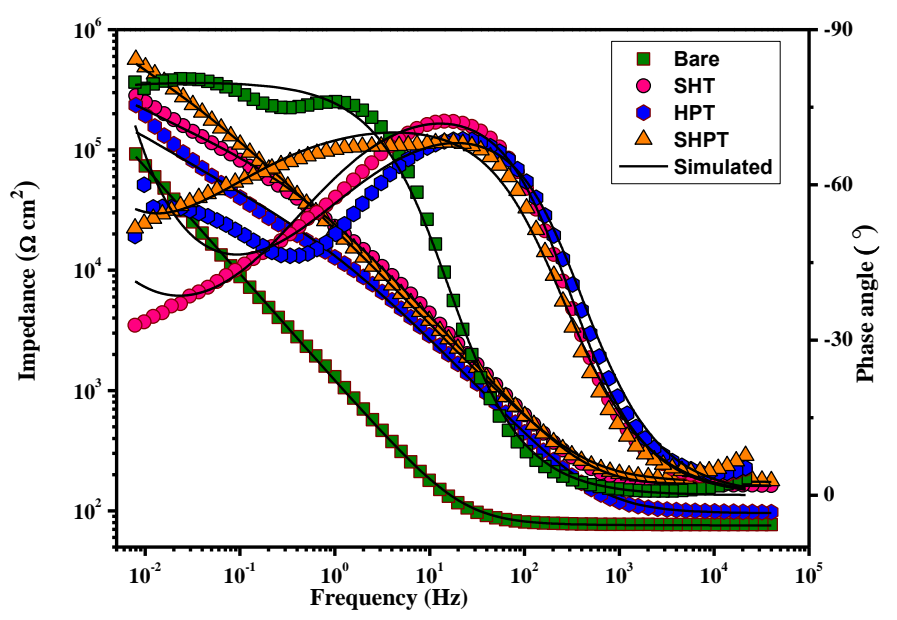

Figure 7. Bode plots of treated specimens in SBF medium.

All the investigated TNZ specimens showed a large capacitive arc in the Nyquist plots, representing the single relaxation time behavior in the investigated frequency range. In general, the diameter of the capacitive arc in the Nyquist plot is an indication of corrosion-resistant performance, and a larger 
diameter denotes enhanced surface resistance against corrosion damage [34]. Comparing the Nyquist plots of bare and treated specimens, it is evident that the diameter of the capacitive arc increased; specifically, the largest diameter was observed for SHPT specimens, revealing the improved barrier performance of the formed layer during surface treatment.

Figure 7 presents the Bode plots of bare and treated TNZ specimens. No changes were observed in the impedance modulus $(\mid \mathrm{ZI})$ at higher frequencies $\left(10^{5}-10^{3} \mathrm{~Hz}\right)$ due to the resistance offered by electrolytic species. On the other hand, the impedance response in the middle frequencies gives a linear relationship with a slope of almost -1 , indicating the capacitive response of the treated TNZ surface. Generally, the $(|Z|)$ value at $0.01 \mathrm{~Hz}$ is utilized to obtain an indication of the barrier performance of an investigated material [22,35]. For the bare specimen, the $|\mathrm{Z}|$ value at $0.01 \mathrm{~Hz}$ was found to be $80.16 \mathrm{k} \Omega \cdot \mathrm{cm}^{2}$ in SBF medium. After surface modification, the $|\mathrm{Z}|$ values for SHT, HPT and SHPT were observed to be $297.29,222.69$ and $605.62 \mathrm{k} \Omega \cdot \mathrm{cm}^{2}$, respectively.

To validate the obtained EIS data for bare and treated TNZ specimens, equivalent circuit fitting analyses were performed using suitable equivalent circuit (EC) models (Figure S3 in the supplementary materials), and the derived EIS parameters are summarized in Table 3.

Table 3. EIS parameters of bare and treated TNZ specimens.

\begin{tabular}{|c|c|c|c|c|c|c|c|}
\hline Substrate & $\begin{array}{c}R_{\mathrm{s}} \\
\left(\Omega \cdot \mathrm{cm}^{2}\right)\end{array}$ & $\begin{array}{c}R_{\mathrm{ct}} \\
\left(\mathrm{k} \Omega \cdot \mathrm{cm}^{2}\right)\end{array}$ & $\begin{array}{c}\mathrm{CPE}_{\mathrm{dl}} \\
\left(\mu \mathrm{F} \cdot \mathrm{cm}^{-2}\right)\end{array}$ & $n_{\mathrm{dl}}$ & $\begin{array}{c}R_{\mathrm{f}} \\
\left(\mathrm{k} \Omega \cdot \mathrm{cm}^{2}\right)\end{array}$ & $\begin{array}{c}\mathrm{CPE}_{f} \\
\left(\mu \mathrm{F} \cdot \mathrm{cm}^{-2}\right)\end{array}$ & $n_{\mathrm{f}}$ \\
\hline Bare & 81.24 & 80.16 & 75.41 & 0.94 & - & - & - \\
\hline SHT & 78.95 & 297.29 & 1.95 & 0.96 & 12.39 & 14.74 & 0.95 \\
\hline HPT & 80.22 & 222.69 & 2.53 & 0.95 & 11.12 & 18.63 & 0.94 \\
\hline SHPT & 19.87 & 605.62 & 0.17 & 0.97 & 18.94 & 0.91 & 0.96 \\
\hline
\end{tabular}

In these circuits, $R_{\mathrm{s}}, R_{\mathrm{f}}$ and $R_{\mathrm{ct}}$ signify the electrolytic, film and charge transfer resistance, respectively. $C P E_{\mathrm{dl}}$ and $\mathrm{CPE}_{\mathrm{f}}$ indicate the constant phase element of the double layer and film capacitance, respectively, which are employed to precisely fit the nonideal capacitive behavior due to the presence of defects and pores in the titanate layer. The exponent $(n)$ of the constant phase element designates the deviance from perfect dielectric performance. When $n$ approaches unity, the coated substrate is close to ideal capacitor behavior [36,37].

From Table 3, the $R_{\mathrm{ct}}$ values of the treated specimens are higher than that of the bare specimen, demonstrating the improved corrosion-resistant performance of the formed layer. In particular, SHPT specimens showed higher $R_{\mathrm{ct}}$ values, corroborating the beneficial role of the mixture of $\mathrm{NaOH}$ and $\mathrm{H}_{2} \mathrm{O}_{2}$ in forming a homogeneous and more compact surface with fewer defects [38]. In addition, the treated specimens showed a decrease in $\mathrm{CPE}_{\mathrm{dl}}$ by two orders of magnitude, indicating the effective hindrance of the penetration of aggressive species $\left(\mathrm{Cl}^{-}\right)$from the SBF medium [39]. Hence, the beneficial role of surface treatment, especially the improvement in SHPT over the TNZ specimen in terms of in vitro corrosion resistant behavior, is reflected in the higher capacitive arcs, higher $R_{\mathrm{ct}}$, lower $\mathrm{CPE}_{\mathrm{dl}}$, highest $E_{\text {corr }}$ and lower $I_{\text {corr }}$ values. The obtained results confirm that the formation of a homogeneous and compact titanate layer improves the corrosion-resistant behavior of the TNZ surface in SBF medium.

\subsection{In Vitro Characterization in SBF Medium}

To estimate the in vitro bioactivity of treated TNZ specimens, the surface morphologies of treated specimens after immersion in SBF medium for 3, 5 and 7 days were determined and are displayed in Figure 8. In a previous study, we evaluated the growth of hydroxyapatite on bare TNZ specimens and reported that $\mathrm{Ca}_{3}\left(\mathrm{PO}_{4}\right)_{2}$ was formed on the entire TNZ surface after 14 days of exposure in SBF medium [16]. Herein, after 3 days of immersion in SBF, all of the treated TNZ specimens exhibited a uniform distribution of globular crystallites covering the entire surface. This growth was enhanced with prolonged immersion time, and finally, some agglomeration of particles was observed after 7 days of immersion in SBF medium. 

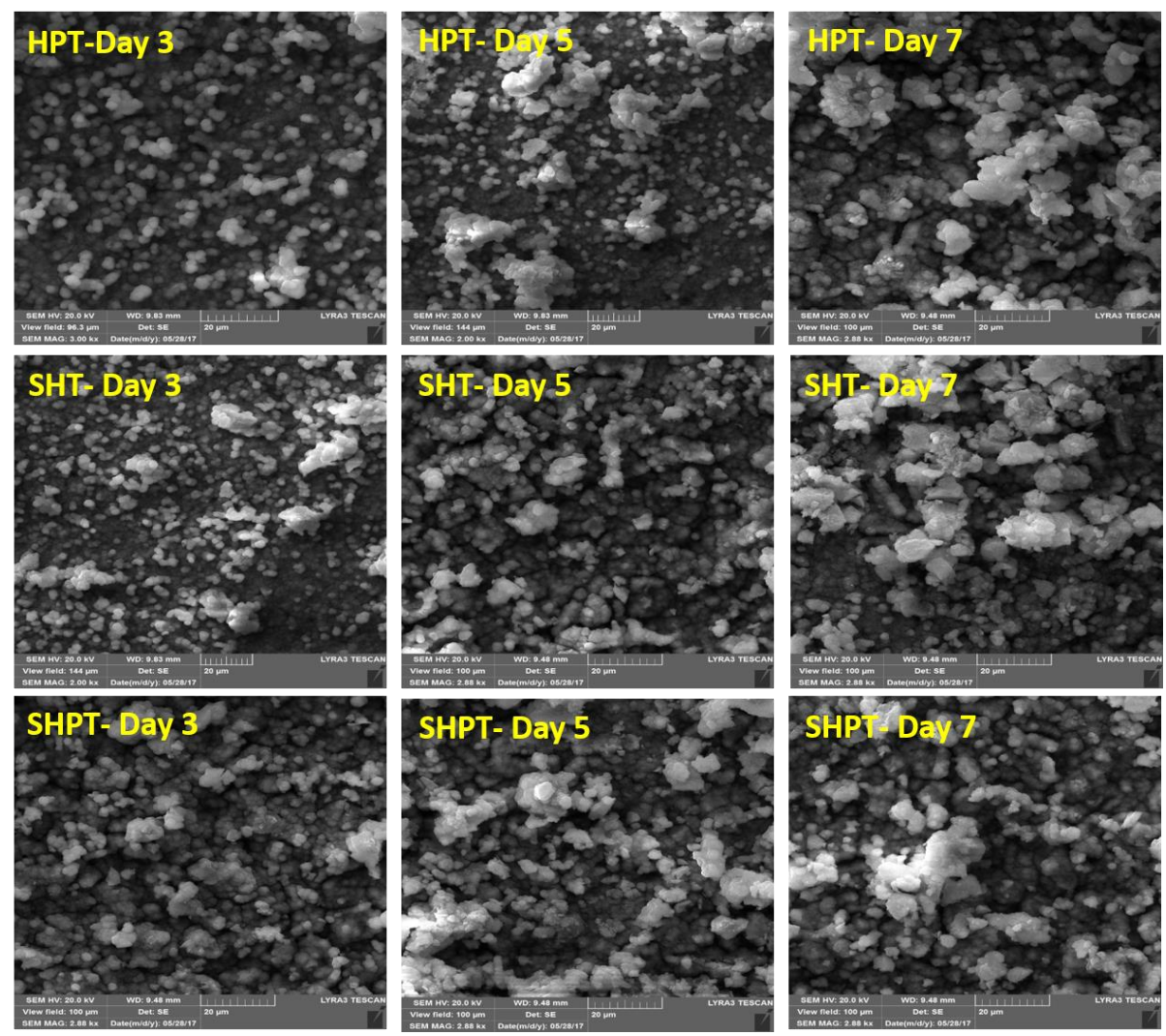

Figure 8. SEM images of treated specimens after immersion in SBF medium.

It has already been established that the formation of apatite occurs through ion exchange between the formed sodium titanate layer and SBF solution, and the mechanism and growth of apatite on treated Ti alloy have been well discussed in the literature [40,41]. Absorbed $\mathrm{OH}^{-}$ions on the treated TNZ surface could considerably enhance the possible apatite formation on the alloy surface. Released sodium ions from the sodium titanate film increased the local $\mathrm{pH}$ near the specimen surface. The negative charges from $\mathrm{HTiO}_{3}{ }^{-}$probably attracted $\mathrm{Ca}^{2+}\left(\right.$ or $\left.\mathrm{Ca}^{2+}\right)$ ions by Coulombic force and $\mathrm{HPO}_{4}{ }^{2-}$ ions by hydrogen bonds, causing the ions to accumulate near the Ti surface. This accumulation of ions enhanced the local supersaturation degree with respect to apatite. When apatite nuclei were formed, they might spontaneously grow by consuming $\mathrm{Ca}^{2+}, \mathrm{HPO}_{4}{ }^{2-}$ and $\mathrm{OH}^{-}$ions from the $\mathrm{SBF}$ solution [42]. Although all the treated TNZ specimens stimulated the nucleation of apatite within 3 days of exposure in SBF, the density and homogeneous distribution of apatite formed in specimens was determined to increase in the order of HPT, SHT and SHPT. The surface treatment of Ti-13Nb-13Zr alloy using anodization, alkali and $\mathrm{H}_{2} \mathrm{O}_{2}$ treatment, etc., efficiently stimulated the attachment of $\mathrm{Ca}^{2+}$ and $\mathrm{HPO}_{4}{ }^{2-}$ ions or the formation of apatite, resulting in enhanced corrosion protection behavior and osseointegration capability [43-45]. The chemical composition of the obtained layer was analyzed using EDS analysis, and the results are displayed in Figure $\mathrm{S} 4$ in the supporting information. The presence of $\mathrm{Ca}, \mathrm{P}$ and $\mathrm{O}$ together with the main alloying elements of TNZ specimens ( $\mathrm{Nb}$ and $\mathrm{Zr}$ ) was observed in the EDS spectra of treated TNZ specimens after immersion in SBF. In addition, the ratios of $\mathrm{Ca} / \mathrm{P}$ for the treated TNZ specimens after exposure for 3,5, and 7 days were found to be 1.53, 1.61, and 1.65, respectively. An increase in $\mathrm{Ca} / \mathrm{P}$ ratio is usually related to the transformation of dicalcium phosphate (DCPA) into hydroxyapatite (HA) [46].

\section{Conclusions}

Surface treatment using chemicals and a thermal route was performed on newly developed TNZ specimens to improve their in vitro corrosion resistance and bioactivity in SBF medium. Surface 
structure and microstructure characterization results from surface profilometry, SEM/EDS, and XRD implied that the SHT and SHPT specimens are characterized by a more compact sodium titanate layer on the TNZ surface, whereas the HPT specimen showed a porous network titania layer with higher surface roughness. The WCA results indicated the improved surface hydrophilicity of the treated TNZ surfaces, which may favor the in vitro bioactivity of these specimens. The in vitro corrosion performance of treated specimens in SBF medium is reflected in the higher capacitive arcs, higher $R_{\mathrm{ct}}$ values, lower $\mathrm{CPE}_{\mathrm{dl}}$, higher $E_{\text {corr }}$ and lower $I_{\text {corr }}$. This observation validates that the formation of a homogeneous and more compact titanate layer improves the corrosion-resistant behavior of TNZ surfaces in SBF medium. Surface characterization of the treated specimens after immersion in SBF medium indicated the uniform distribution of hydroxyapatite particles on the treated TNZ specimens, which validated their in vitro bioactive performance in SBF medium.

Supplementary Materials: The following are available online at http://www.mdpi.com/2079-6412/9/5/344/s1, Figure S1: EDS analysis of bare and surface treated TNZ specimens, Table S1: Surface roughness parameters of bare and treated TNZ specimens, Figure S2: LPR curves of (a) Bare, (b) SHT, (c) HPT and (d) SHPT, Figure S3: EIS circuit models of (a) Bare and (b) treated TNZ substrates, Figure S4: EDS results of bare and surface treated substrates.

Author Contributions: Conceptualization, M.K.A. and N.A.-A.; Methodology, M.K.A.; Software, M.K.A.; Validation, M.K.A., M.A.H. and N.A.-A.; Formal Analysis, M.K.A.; Investigation, M.K.A. M.A.H. and A.Y.A.; Resources, M.K.A., M.A.H. and N.A.-A.; Data Curation, M.K.A. and A.Y.A.; Writing-Original Draft Preparation, M.K.A.; Writing-Review and Editing, M.K.A., M.A.H. and N.A.-A.; Supervision, N.A.-A.; Project Administration, N.A.-A.; Funding Acquisition, M.A.H. and N.A.-A.

Funding: This research was funded by KACST-NSTIP via Project No. \# 15-ADV4632-04.

Acknowledgments: The authors acknowledge the support provided by King Fahd University of Petroleum \& Minerals, Dhahran, Saudi Arabia.

Conflicts of Interest: There is no potential conflict of interest.

\section{References}

1. Eisenbarth, E.; Velten, D.; Müller, M.; Thull, R.; Breme, J. Biocompatibility of $\beta$ stabilizing elements of titanium alloys. Biomaterials 2004, 25, 5705-5713. [CrossRef]

2. Niinomi, M. Recent research and development in titanium alloys for biomedical applications and healthcare goods. Sci. Technol. Adv. Mater. 2003, 4, 445-454. [CrossRef]

3. Geetha, M.; Singh, A.K.; Asokamani, R.; Gogia, A.K. Ti based biomaterials, the ultimate choice for orthopaedic implants-A review. Prog. Mater. Sci. 2009, 54, 397-425. [CrossRef]

4. Chen, Q.; Thouas, G.A. Metallic implant biomaterials. Mater. Sci. Eng. R Rep. 2015, 87, 1-57. [CrossRef]

5. Asri, R.; Harun, W.; Samykano, M.; Lah, N.; Ghani, S.; Tarlochan, F.; Raza, M.A. Corrosion and surface modification on biocompatible metals: A review. Mater. Sci. Eng. C 2017, 77, 1261-1274. [CrossRef]

6. Rafieerad, A.R.; Ashra, M.R.; Mahmoodian, R.; Bushroa, A.R. Surface characterization and corrosion behavior of calcium phosphate-base composite layer on titanium and its alloys via plasma electrolytic oxidation: A review paper. Mater. Sci. Eng. C 2015, 57, 397-413. [CrossRef]

7. Mohammed, M.T.; Khan, Z.K.; Siddique, A.N. Surface modifications of titanium materials for developing corrosion behavior in human body environment: A review. Procedia Mater. Sci. 2014, 6, 1610-1618. [CrossRef]

8. Hussein, M.A.; Mohammed, A.S.; Al-Aqeeli, N. Wear characteristics of metallic biomaterials: A review. Materials 2015, 8, 2749-2768. [CrossRef]

9. Geetha, M.; Dhinasekaran, D.; Rajamanickam, A. Biomedical implants: Corrosion and its prevention-A review. Recent Pat. Corros. Sci. 2010, 2, 40-54.

10. Butev, E.; Esen, Z.; Bor, S. In vitro bioactivity investigation of alkali treated Ti6Al7Nb alloy foams. Appli. Sur. Sci. 2015, 327, 437-443. [CrossRef]

11. Shukla, A.K.; Balasubramaniam, R. Effect of surface treatment on electrochemical behavior of CPTi, Ti-6Al-4V and $\mathrm{Ti}-13 \mathrm{Nb}-13 \mathrm{Zr}$ alloys in simulated human body fluid. Corros. Sci. 2006, 48, 1696-1720. [CrossRef]

12. Tamilselvi, S.; Raghavendran, H.B.; Srinivasan, P.; Rajendran, N. In vitro and in vivo studies of alkali- and heat-treated Ti-6Al-7Nb and Ti-5Al-2Nb-1Ta alloys for orthopedic implants. J. Biomed. Mater. Res. A 2009, 90, 380-386. [CrossRef] [PubMed] 
13. Karthega, M.; Nagarajan, S.; Rajendran, N. In vitro studies of hydrogen peroxide treated titanium for biomedical applications. Electrochim. Acta 2010, 55, 2201-2209. [CrossRef]

14. Kim, H.M.; Miyaji, F.; Kokubo, T.; Nakamura, T. Effect of heat treatment on apatite forming ability of Ti metal induced by alkali treatment. J. Mater. Sci. Mater. Med. 1997, 8, 341-347. [CrossRef]

15. Hussein, M.A.; Suryanarayana, C.; Al-Aqeeli, N. Fabrication of nano-grained Ti-Nb-Zr biomaterials using spark plasma sintering. Mater. Des. 2015, 87, 693-700. [CrossRef]

16. Hussein, M.; Kumar, M.; Drew, R.; Al-Aqeeli, N. Electrochemical corrosion and in vitro bioactivity of nano-grained biomedical Ti-20Nb-13Zr alloy in a simulated body fluid. Materials 2018, 11, 26. [CrossRef] [PubMed]

17. Hussein, M.A.; Al-Aqeeli, N. Titanium Alloys for Biomedical Applications and Fabrication Methods Thereof. U.S. Patent 9828655B2, 28 November 2017.

18. Wang, X.J.; Li, Y.C.; Xiong, J.Y.; Hodgson, P.D.; Wen, C.E. Porous TiNbZr alloy scaffolds for biomedical applications. Acta Biomater. 2009, 5, 3616-3624. [CrossRef]

19. Karthega, M.; Rajendran, N. Formation of nanoporous oxide layer over a binary $\beta$-phase titanium in simulated body fluid. J. Porous Mater. 2012, 19, 573-577. [CrossRef]

20. Kumar, A.M.; Sudhagar, P.; Ramakrishna, S.; Kang, Y.S.; Kim, H.; Gasem, Z.M.; Rajendran, N. Evaluation of chemically modified Ti-5Mo-3Fe alloy surface: Electrochemical aspects and in vitro bioactivity on MG63 cells. Appli. Surf. Sci. 2014, 307, 52-61. [CrossRef]

21. Gao, Z.; Li, Q.; He, F.; Huang, Y.; Wan, Y. Mechanical modulation and bioactive surface modification of porous Ti-10Mo alloy for bone implants. Mater. Des. 2012, 42, 13-20. [CrossRef]

22. Sasikumar, Y.; Rajendran, N. Influence of surface modification on the apatite formation and corrosion behavior of Ti and Ti-15Mo alloy for biomedical applications. Mater. Chem. Phy. 2013, 138, 114-123. [CrossRef]

23. Kumar, A.M.; Hussein, M.A.; Adesina, A.Y.; Ramakrishna, S.; Al-Aqeeli, N. Influence of surface treatment on PEDOT coatings: Surface and electrochemical corrosion aspects of newly developed Ti alloy. RSC Adv. 2018, 8, 19181-19195. [CrossRef]

24. Lin, L.; Wang, H.; Ni, M.; Rui, Y.; Cheng, T.Y.; Cheng, C.K.; Pan, X.; Li, G.; Lin, C. Enhanced osseointegration of medical titanium implant with surface modifications in micro/nanoscale structures. J. Ortho. Trans. 2014, 2, 35-42.

25. Lai, Y.K.; Lin, C.J.; Wang, H.; Huang, J.Y.; Zhuang, H.F.; Sun, L. Superhydrophilic/ superhydrophobic micro pattern on $\mathrm{TiO}_{2}$ nanotube films by photocatalytic lithography. Electrochem. Commun. 2008, 10, 387-391. [CrossRef]

26. Bo, A.; Zhan, H.; Bell, J.; Zhu, H.; Gu, Y. Mechanical bending properties of sodium titanate nanowires. RSC Adv. 2014, 4, 56970-56976. [CrossRef]

27. Karthega, M.; Rajendran, N. Hydrogen peroxide treatment on Ti-6Al-4V alloy: A promising surface modification technique for orthopaedic application. Appli. Sur. Sci. 2010, 256, 2176-2183. [CrossRef]

28. Wu, W.; Nancollas, G.H. Kinetics of heterogeneous nucleation of calcium phosphates on anatase and rutile. J. Colloid Interface Sci. 1998, 199, 206-211. [CrossRef]

29. Kumar, A.M.; Adesina, A.Y.; Hussein, M.A.; Ramakrishna, S.; Al-Aqeeli, N.; Akhtar, S.; Saravanan, S. PEDOT/FHA nanocomposite coatings on newly developed Ti-Nb-Zr implants: Biocompatibility and surface protection against corrosion and bacterial infections. Mater. Sci. Eng. C 2019, 98, 482-495. [CrossRef]

30. Kilpadi, D.V.; Lemons, J.E. Surface energy characterization of unalloyed Ti implants. J. Biomed. Mater. Res. 1994, 28, 1419-1425. [CrossRef]

31. Pesskova, V.; Kubies, D.; Hulejova, H.; Himmlova, L. The influence of implant surface properties on cell adhesion and proliferation. J. Mater. Sci. Mater. Med. 2007, 18, 465-473. [CrossRef]

32. Chelariu, R.; Bolat, G.; Izquierdo, J.; Mareci, D.; Gordin, D.M.; Gloriant, T.; Souto, R.M. Metastable beta Ti-Nb-Mo alloys with improved corrosion resistance in saline solution. Electrochim. Acta 2014, 137, 280-289. [CrossRef]

33. Hussein, M.A.; Azeem, M.; Kumar, A.M.; Al-Aqeeli, N.; Ankah, N.K.; Sorour, A.A. Influence of thermal treatment on the microstructure, mechanical properties, and corrosion resistance of newly developed Ti20Nb13Zr biomedical alloy in a simulated body environment. J. Mater. Eng. Perform. 2019, 28, 1337-1350. [CrossRef] 
34. Hussein, M.A.; Kumar, A.M.; Yilbas, B.S.; Al-Aqeeli, N. laser nitriding of the newly developed Ti-20Nb-13Zr at.\% biomaterial alloy to enhance its mechanical and corrosion properties in simulated body fluid. J. Mater. Eng. Perform. 2017, 26, 5553-5562. [CrossRef]

35. Akhtar, S.; Matin, A.; Kumar, A.M.; Ibrahim, A.; Laoui, T. Enhancement of anticorrosion property of 304 stainless steel using silane coating. Appli. Surf. Sci. 2018, 440, 286-1297. [CrossRef]

36. Hussein, M.A.; Yilbas, B.; Kumar, A.M.; Drew, R.; Al-Aqeeli, N. Influence of Laser nitriding on the surface and corrosion properties of Ti-20Nb-13Zr alloy in artificial saliva for dental applications. J. Mater. Eng. Perform. 2018, 27, 4655-4664. [CrossRef]

37. Kumar, A.M.; Babu, R.S.; Obot, I.B.; Adesina, A.Y.; Ibrahim, A.; de Barros, A.L.F. Promising hard carbon coatings on $\mathrm{Cu}$ substrates: Corrosion and tribological performance with theoretical aspect. J. Mater. Eng. Perform. 2018, 27, 2306-2316. [CrossRef]

38. Sasikumar, Y.; Rajendran, N. Surface modification and in vitro characterization of Cp-Ti and Ti-5Al-2Nb-1Ta alloy in simulated body fluid. J. Mater. Eng. Perform. 2012, 21, 2177-2187. [CrossRef]

39. Chen, X.B.; Li, Y.C.; Hodgson, P.D.; Wen, C. The importance of particle size in porous titanium and nonporous counterparts for surface energy and its impact on apatite formation. Acta Biomater. 2009, 5, 2290-2302. [CrossRef]

40. Chen, X.B.; Li, Y.C.; Plessis, J.D.; Hodgson, P.D.; Wen, C.E. Influence of calcium ion deposition on apatite-inducing ability of porous titanium for biomedical applications. Acta Biomater. 2009, 5, 1808-1820. [CrossRef]

41. Xiong, J.; Li, Y.; Wang, X.; Hodgson, P.; Wen, C.E. Mechanical properties and bioactive surface modification via alkali-heat treatment of a porous Ti- $18 \mathrm{Nb}-4 \mathrm{Sn}$ alloy for biomedical applications. Acta Biomater. 2008, 4, 1963-1968. [CrossRef]

42. Simka, W. Preliminary investigations on the anodic oxidation of Ti-13Nb-13Zr alloy in a solution containing calcium and phosphorus. Electrochim. Acta 2011, 56, 9831-9837. [CrossRef]

43. Pan, J.; Liao, H.; Leygraf, C.; Thierry, D.; Li, J. Variation of oxide films on titanium induced by osteoblast-like cell culture and the influence of an $\mathrm{H}_{2} \mathrm{O}_{2}$ pretreatment. J. Biomed. Mater. Res. 1998, 40, 244-256. [CrossRef]

44. Baker, M.A.; Assis, S.L.; Grilli, R.; Costa, I. Investigation of the electrochemical behavior and surface chemistry of a Ti-13Nb-13Zr alloy exposed in MEM cell culture media with and without the addition of $\mathrm{H}_{2} \mathrm{O}_{2}$. Surf. Interface Anal. 2008, 40, 220-224. [CrossRef]

45. Sasikumar, Y.; Kumar, A.M.; Babu, R.S.; Rahman, M.M.; Samyn, L.M.; de Barros, A.L.F. Biocompatible hydrophilic brushite coatings on AZX310 and AM50 alloys for orthopaedic implants. J. Mater. Sci. Mater. Medi. 2018, 29, 123-137. [CrossRef] [PubMed]

46. Kumar, A.M.; Hassan, S.F.; Sorour, A.A.; Paramsothy, M.; Gupta, M. Investigation on the controlled degradation and in vitro mineralization of carbon nanotube reinforced AZ31 nanocomposite in simulated body fluid. Met. Mater. Inter. 2019, 25, 105-116. [CrossRef] 